\title{
An ECCM scheme for multi-false targets jamming based on orthogonal diversity
}

\author{
Wen-Dong Chen ${ }^{\dagger}$ and Ya-Qiong Yan, \\ The second Academy of China Aerospace Science and Industry Corp. \\ Beijing, 100854, China \\ E-mail: chen_eecs@sina.com \\ Zhi-Yi He \\ Beijing Institute of Remote Sensing Equipment, Beijing, 100854, China \\ E-mail:Hzy25147@sina.com
}

\begin{abstract}
According to the initial value sensitivity of chaos-based frequency modulation signals and Doppler-delay coupling characteristic of chirp signals, two kinds of orthogonal pulse diversity signals are proposed for suppressing multi-false targets jamming produced by DRFM repeat jammer in this work. With the application of self-adaption switchover of the diversity techniques, the presented method can update the strategy automatically on the basis of different DRFM modes. Besides, quantitative analysis and verification of the range-Doppler image and ECCM performance of new method are obtained through using Monte Carlo simulation and the results are proved to be consistent with theoretic analysis.

Keywords: Orthogonal Diversity; Multi-False Targets Jamming; Radar ECCM; DRFM.
\end{abstract}

\section{Introduction}

The repeat jammer based on digital radio frequency memory[1](DRFM) techniques captured radar signals and retransmitted after noise convolution modulation. The jamming signals are compressed by the radar system and generated multi-false targets jamming[2]. Such kind of jamming signals has been possessed with characteristics of pulse compression of real echo signal and phase coherence. It can ignore side-lobe cancellation (SLC) and side-lobe blanking(SLB) technique of the new system radar, entering the radar receiver from the radar antenna main-lobe or side-lobe. Besides, pulse compressing gain of the radar receiver itself is applied to generate the dense multi-false targets in the range and Doppler dimension to achieve the deception and suppression jamming of the new system of radar[3].

In current days, the study of multi-false targets jamming is mainly concentrates on the generation and recognition methods. There remain few research results of the relevant countermeasures. In [4], waveform orthogonal 
diversity (WOD) theory is used for the suppression of active deception jamming. In addition, the multi-tone phase-modulated (MTPM) LFM signal and slopevarying (SV) LFM signal are proposed in [5]. Through using the mismatched relationship between the jamming signal and the echo signal, false targets can be eliminated by thresholds. In[6], filtering method is resorted to replacing the threshold method for making the improvement of [5].

In this paper, two kinds of waveform orthogonal diversity method with the application of chaos-based frequency modulation (CBFM) signal and frequency and slope varying LFM (FSVLFM) signal are used to multi-false targets jamming environments and an adaptive ECCM scheme is proposed according to different work modes of DRFM repeat jammer. The structure is organized as follows: section 2 presents the signal model of orthogonal signals and echo signals in multi-false targets jamming environments. Section 3 establishes the ECCM scheme based on WOD and adaptive switchover method, and performs Monte Carlo simulation in Section 4. Finally, section 5 draws a conclusion and points out the further work.

\section{The Signal Model}

\subsection{CBFM signaling}

The continuous pulse CBFM signal is defined by[7]

$$
s_{\mathrm{CBFM}}(t)=A \exp \left[\mathrm{j} 2 \pi K \int_{0}^{t} \varphi(\tau) \mathrm{d} \tau\right] s_{0}(t) \quad 0 \leq t \leq T
$$

Where $s_{0}(t)$ is the carrier signal; $K$ is the frequency modulation index; $\varphi(t)$ is the continuous chaotic signal defined by

$$
\varphi(t)=\sum_{n=0}^{N} x_{n} \operatorname{sinc}\left(\frac{t}{\triangle t}-n\right)
$$

where $x_{n}$ is a discrete chaotic sequence, and $\Delta t$ is a sampling interval.

The instantaneous frequency of CBFM signal has the following form:

$$
f=\frac{\mathrm{d} \Phi(\mathrm{t})}{\mathrm{d} t}=\frac{\mathrm{d} K \int_{0}^{t} \varphi(\tau) \mathrm{d} \tau}{\mathrm{d} t}=K \varphi(t) \quad 0 \leq t \leq T
$$

It can be deduced that the frequency modulation bandwidth of CBFM signal is:

$$
K \varphi_{\min }(t) \leq \Delta f \leq K \varphi_{\max }(t)
$$

The sampling rate must meet Nyquist sampling, which is 


$$
f_{s}=\frac{1}{\Delta t} \geq 2 K \varphi_{\max }(t)=2 K \max \left[\sum_{n=0}^{N} x_{n} \operatorname{sinc}\left(\frac{t}{\Delta t}-n\right)\right]=2 K \max \left(x_{n}\right)
$$

CBFM signal has the property of initial value sensitivity, and the two signals with different initial values have the orthogonal characteristics ${ }^{[7]}$. According to the different types of chaotic maps, the following three kinds of common CBFM signals can be found.

The Bernoulli mapping is defined as:

$$
x_{\mathrm{B}}(n+1)=\mu\left[0.25-x_{\mathrm{B}}^{2}(n)\right]-0.5 \quad x_{\mathrm{B}}(n) \in[-0.5,0.5]
$$

where $\mu \in(1,2]$.Substituted Eq. (6) into Eq. (1) and make discretization, the obtained Bernoulli CBFM can be defined by

$$
s_{\mathrm{B}-\mathrm{CBFM}}(n)=A \exp \left[\mathrm{j} 2 \pi K \sum_{n=0}^{N} x_{\mathrm{B}}(n) \triangle t\right] s_{0}(n)
$$

It can be deducted from Eq. (5) that

$$
f_{s}=\frac{1}{\Delta t} \geq 2 K \max \left(x_{n}\right)=K
$$

Substituting Eq. (7), that is

$$
s_{\mathrm{B}-\mathrm{CBFM}}(n)=A \exp \left[\sum_{n=0}^{N} \mathrm{j} 2 \pi x_{\mathrm{B}}(n)\right] s_{0}(n)
$$

Auto-correlation function (ACF) of Bernoulli CBFM and cross-correlation function $(\mathrm{CCF})$ with different initial values are presented in Fig.1 (a).

The Chebyshev mapping is defined as follows

$$
x_{\mathrm{C}}(n+1)=0.5 \cos \left\{\mu \arccos \left[2 x_{\mathrm{C}}(n)\right]\right\} \quad x_{\mathrm{C}}(n) \in[-0.5,0.5]
$$

where $\mu \in(1,10]$. Substituted Eq. (10) into Eq. (1) and make discretization to satisfy Eq. (5), that is

$$
s_{\mathrm{C}-\mathrm{CBFM}}(n)=A \exp \left[\mathrm{j} 2 \pi \sum_{n=0}^{N} x_{\mathrm{C}}(n)\right] s_{0}(n)
$$

The ACF and CCF of Chebyshev CBFM are shown in Fig.1 (b).

The Tent mapping is defined by

$$
x_{\mathrm{T}}(n+1)=0.5-\mu\left|x_{\mathrm{T}}(n)\right| \quad x_{\mathrm{T}}(n) \in[-0.5,0.5]
$$

where $\mu \in(1,2]$. Substituted Eq. (12) into Eq. (1) and discretized to satisfy Eq. (5), that is 


$$
s_{\mathrm{T}-\mathrm{CBFM}}(n)=A \exp \left[\mathrm{j} 2 \pi \sum_{n=0}^{N} x_{\mathrm{T}}(n)\right] s_{0}(n)
$$

The ACF and CCF of Tent CBFM are as shown in Fig.1 (c).

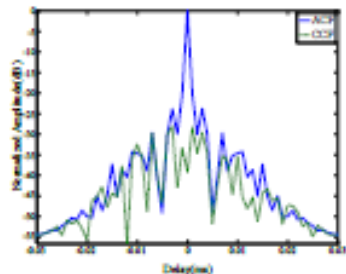

(a) Bernulli CBFM



(b) Chebyshev CBFM

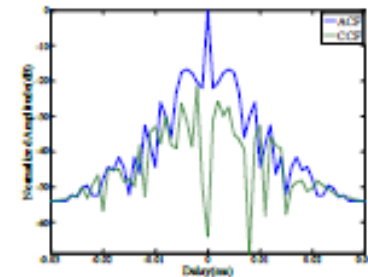

(c) Tent CBFM

Fig. 1.The ACF and CCF of CBFM signals

\subsection{FSVLFM signaling}

FSVLFM signal is defined by

$$
s_{\mathrm{FSVLF}_{m}}(t)=\exp \left[\mathrm{j}\left(2 \pi \alpha_{m} t+\pi \beta_{m} t^{2}\right)\right] \quad 0 \leq t \leq T
$$

where $m$ is for pulse number; $\alpha_{m}$ represents frequency parameters and $\beta_{m}$ represents slope varying parameters.

LFM signal has Doppler-delay coupling characteristic, and the target Doppler frequency will be coupled to the time delay after pulse compression. The Doppler-delay coupling relationship is as follows

$$
\beta \tau+\xi=0
$$

where $\tau$ is for time delay, $\xi$ is for Doppler Shift, $\beta=B / T$ is for frequency modulation rate ( $B$ is for bandwidth, $T$ is for pulse width). For pulse radar, when receiving the echoes, generally, only the echo data within range gate is intercepted to extract target information. Therefore, when $\alpha_{m} \neq \alpha_{n}$ and the frequency difference between the two signals reaches a certain range, after pulse compression, the peak point will fall outside the range gate, leading to being equivalent to the two orthogonal signals. Assuming that the range gate width is $T_{\mathrm{G}}$, when the jump frequency $\Delta f$ between adjacent pulse signals satisfies Eq. (16), it indicates that the two signals are orthogonal.

$$
\triangle f=\left|\alpha_{m}-\alpha_{n}\right| \geq \beta T_{\mathrm{G}}
$$

When $\alpha_{m}=\alpha_{n}=\alpha$ and $\beta_{m} \neq \beta_{n}$, the CCF is still LFM signal ${ }^{[5]}$, and meets the orthogonality, which is 


$$
\exp \left(\mathrm{j} \pi \frac{\beta_{m} \beta_{n}}{\beta_{m}-\beta_{n}} t^{2}\right)
$$

The ACF and CCF of FSVLFM signal are as presented in Fig. 2. According to Fig.2, with the selection of the appropriate frequency and slope parameters, the CCF meets orthogonal requirements.

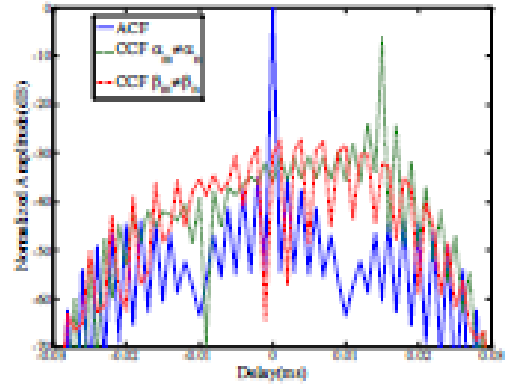

(a) $\alpha_{m} \neq \alpha_{n}: \beta_{m} \neq \beta_{n}$

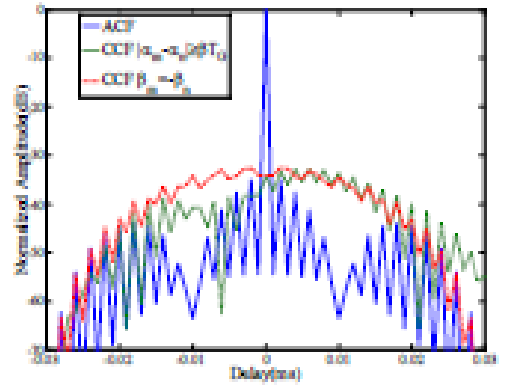

(b) $\left|a_{m}-a_{n}\right| \geqslant \beta T_{G} ; \quad \beta_{m}=-\beta_{n}=\beta$

Fig. 2.The ACF and CCF of FSVLFM signal

\subsection{Echo signaling with multi-false targets jamming}

Under the condition of ECM environments, the echo signal can be expressed as

$$
\begin{aligned}
s_{r}\left(t, u_{m}\right) & =s_{\text {target }}\left(t, u_{m}\right)+s_{\text {jammer }}\left(t, u_{m}\right) \\
& =\sum_{n} \sigma_{n} p_{m}\left[t-t_{n}\left(u_{m}\right)\right]+\sum_{l} \rho_{l} p_{M}\left[t-t_{l}^{\prime}\left(u_{m}\right)\right]
\end{aligned}
$$

where $s_{\text {target }}\left(t, u_{m}\right)$ is the real target echoes, corresponding to $m^{\text {th }}$ pulse and matches with radar transmitted signal; $\sigma_{n}$ is the real target scatter points; $t_{n}$ is the real target corresponding delay; $p_{m}$ is the echo pulse matching with the transmitted pulse; $s_{\text {jammer }}\left(t, u_{m}\right)$ is jamming signal corresponding to the $m^{\text {th }}$ pulse; $\rho_{l}$ is the multi-false targets jamming range profile; $t_{l}^{\prime}$ is the time delay of jamming signal, and $p_{M}$ is the jamming signal retransmitted by the jammer.

\section{ECCM Scheme Based on Orthogonal Diversity Design}

\subsection{DRFM forwarding mode}

In general, DRFM jammer uses all-pulse storage mode for the modulation signal[6] and the forward signal is lagging behind the radar transmit signal for one or more pulse repetition interval (PRI). Forwarding modes of DRFM can be divided into three types. 
In circular forwarding mode, DRFM jammer transmits the same jamming signal over a period of time, i.e. $M$ in (18) is a fixed value as presented in Table 1 .

Table 1. Circular Forwarding Mode

\begin{tabular}{ccccc}
\hline Pulse number & 1 & 2 & 3 & 4 \\
\hline Transmit signal & $p_{1}(t)$ & $p_{2}(t)$ & $p_{3}(t)$ & $p_{4}(t)$ \\
Jamming signal & $p_{1}(t)$ & $p_{1}(t)$ & $p_{1}(t)$ & $p_{1}(t)$ \\
\hline
\end{tabular}

In one-by-one forwarding mode, DRFM jammer intercepts the radar signal at each PRI and delays the forwarding, that is, $M=m-1$, according to Table 2.

Table 2. One-by-one Forwarding Mode

\begin{tabular}{ccccc}
\hline Pulse number & 1 & 2 & 3 & 4 \\
\hline Transmit signal & $p_{1}(t)$ & $p_{2}(t)$ & $p_{3}(t)$ & $p_{4}(t)$ \\
Jamming signal & $p_{4}(t)$ & $p_{1}(t)$ & $p_{2}(t)$ & $p_{3}(t)$ \\
\hline
\end{tabular}

In broadband forwarding mode, DRFM jammer forwards the broadband modulated signal within a certain bandwidth, showing in Table 3.

Table 3. Broadband Forwarding Mode

\begin{tabular}{ccccc}
\hline Pulse number & 1 & 2 & 3 & 4 \\
\hline Transmit signal & $p_{1}(t)$ & $p_{2}(t)$ & $p_{3}(t)$ & $p_{4}(t)$ \\
Jamming signal & $\left.p_{1}(t)\right|_{B}{ }^{\mathrm{a}}$ & $\left.p_{1}(t)\right|_{B}$ & $\left.p_{1}(t)\right|_{B}$ & $\left.p_{1}(t)\right|_{B}$ \\
\hline
\end{tabular}

Note: Table notes.

$\left.{ }^{\mathrm{a}} p_{1}(t)\right|_{B}$ indicates that $p_{1}(t)$ is broadband modulated within bandwidth $B$.

\subsection{Orthogonal diversity design}

Taking four-pulse diversity as a basis, the ECCM method of CBFM and FSVLFM orthogonal diversity is designed as presented in Table 4. $s_{\mathrm{CBFM}}(t) \mid x_{0}=X_{M}$ denotes chaotic frequency modulation signal with initial value $X_{M} \cdot s_{\mathrm{FSVLFM}}(t) \mid \alpha_{m}=\alpha, \beta_{m}=\beta$ represents FSVLFM signal when $\alpha_{m}=\alpha, \beta_{m}=\beta$, and the selection of $\alpha_{1}, \alpha_{2}$ satisfy Eq.(16).

Table 4. Orthogonal Diversity Design

\begin{tabular}{ccc}
\hline Pulse & CBFM-WOD & FSVLFM-WOD \\
\hline$p_{1}(t)$ & $s_{\mathrm{CBFM}}(t) \mid x_{0}=X_{1}$ & $s_{\mathrm{FSVLFM}}(t) \mid \alpha_{m}=\alpha_{1}, \beta_{m}=\beta$ \\
$p_{2}(t)$ & $s_{\mathrm{CBFM}}(t) \mid x_{0}=X_{2}$ & $s_{\mathrm{FSVLFM}}(t) \mid \alpha_{m}=\alpha_{2}, \beta_{m}=\beta$ \\
$p_{3}(t)$ & $s_{\mathrm{CBFM}}(t) \mid x_{0}=X_{3}$ & $s_{\mathrm{FSVLFM}}(t) \mid \alpha_{m}=\alpha_{1}, \beta_{m}=-\beta$ \\
$p_{4}(t)$ & $s_{\mathrm{CBFM}}(t) \mid x_{0}=X_{4}$ & $s_{\mathrm{FSVLFM}}(t) \mid \alpha_{m}=\alpha_{2}, \beta_{m}=-\beta$ \\
\hline
\end{tabular}

Theoretically, for different DRFM mode, the appropriate diversity signal should be selected accordingly. The FSVLFM signal possesses the best orthogonality for one-by-one forwarding mode, which is because FSVLFM signal maps all of the jamming signals outside the radar range gate to maximize 
the signal-to-jamming ratio (SJR).However, in circular and broadband forwarding mode, the insensitivity of LFM signal to the Doppler frequency shift will degrade SJR. Therefore, the Doppler-insensitive CBFM signal should be selected. As a result, the optimal diversity strategy is as presented in Table 5.

Table 5. Waveform Diversity Strategy

\begin{tabular}{ccc}
\hline DRFM Mode & CBFM-WOD & FSVLFM-WOD \\
\hline Circular forwarding mode & $\sqrt{ }$ & $\times^{\mathrm{a}}$ \\
One-by-one forwarding mode & $\circ^{\mathrm{b}}$ & $\sqrt{ }$ \\
Broadband forwarding mode & $\sqrt{ }$ & $\times$ \\
\hline
\end{tabular}

Note: Table notes.

a $\sqrt{\text { indicates priority. }}$

${ }^{\mathrm{b}} \mathrm{O}$ indicates alternative.

${ }^{c} \times$ indicates inappropriate.

The orthogonal diversity switching model is shown in Fig.3. The traditional receive branch is divided into two parts: the first part is to complete the traditional data processing function [Eq. (19), $1 \leqslant i \leqslant M$ ]; The second part is orthogonal diversity adaptive switching branch, and there is the need for matched filtering of all signals in the diversity group [Eq.(20),
[E $\left.\max \left(N_{1}, N_{2}, \ldots, N_{M}\right)\right]$. Besides, the signal with the maximum threshold points is the signal transmitted by jammer, in continuous comparison of $M$ times with determining jamming mode and waveform diversity strategy is selected through the review of the table (Table 5).

$$
\begin{aligned}
& s_{1}\left(t, u_{m}\right)=s_{r}\left(t, u_{m}\right) * p_{m}^{*}(-t)=s_{\text {target }}\left(t, u_{m}\right) * p_{m}^{*}(-t)+s_{\text {jammer }}\left(t, u_{m}\right) * p_{m}^{*}(-t) \\
& s_{2}\left(t, u_{m}\right)=s_{r}\left(t, u_{m}\right) * p_{i}^{*}(-t)=s_{\text {target }}\left(t, u_{m}\right) * p_{i}^{*}(-t)+s_{\text {jammer }}\left(t, u_{m}\right) * p_{i}^{*}(-t)
\end{aligned}
$$

Fig. 3.Orthogonal diversity switching model

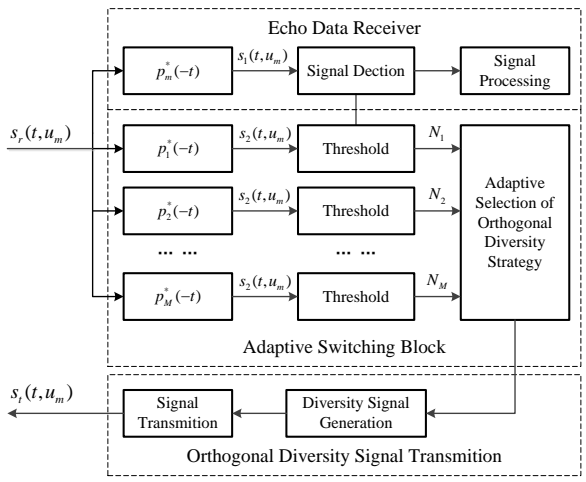




\section{Simulation}

In the simulation, the off-board active radar jamming scenarios is taken as example. Assuming that the true target is a stationary point target, and the DRFM jammer can work in different forwarding modes. Besides, the bandwidth is much larger than the radar system. The key parameters of the radar and the jammer is shown in Table 6.

Table 6. Simulation Key Parameters

\begin{tabular}{ccc}
\hline Device & Parameter Name & Value \\
\hline \multirow{4}{*}{ radar } & Pulse Width(ms) & 0.1 \\
& PRI $(\mathrm{ms})$ & 1 \\
& Bandwidth $(\mathrm{MHz})$ & 20 \\
& Sampling Rate $(\mathrm{MHz})$ & 50 \\
DRFM & Sampling Points in Range Gate & 100 \\
jammer & Coherent Accumulated Pulse Number & 128 \\
& Forwarding Delay(ms) & 1 \\
& Bandwidth(MHz) & 200 \\
\hline
\end{tabular}

\subsection{Impact of CBFM-WOD signal parameter change on SJR}

Through conducting 5000 times Monte Carlo simulation experiments, the impact of CBFM-WOD signal parameter change on SJR is presented in Fig.4.

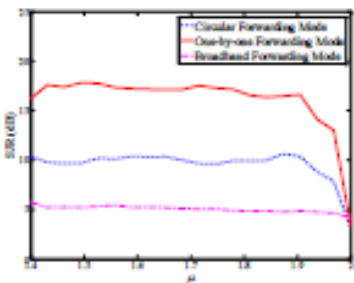

(a) Bemoulli CBFM

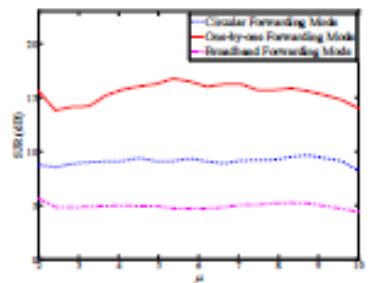

(b) Chebyshev CBFM

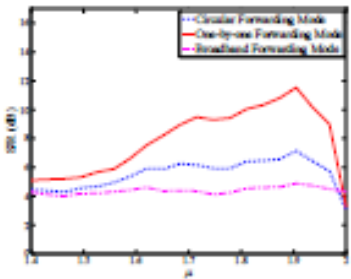

(c)Tent CBFM

Fig. 4.Impact of CBFM-WOD signal parameter change on SJR

According to Fig. 4, when $\mu>1.9$, SJR decreases obviously; $\mu$ relatively has exerted little impact on Chebyshev CBFM signal SJR, and the impact is slightly less when $\mu<4$; for Tent CBFM, when $\mu>1.9$ or $\mu<1.7$, SJR decreases obviously. Therefore, reasonable range of $\mu$ value is presented in Table 7.

Table 7. Range of CBFM-WOD Signal Parameter

\begin{tabular}{cc}
\hline Chaotic Map & $\mu$ \\
\hline Bernulli & $(1.4,1.9]$ \\
Chebyshev & {$[4,10]$} \\
Tent & {$[1.7,1.9]$} \\
\hline
\end{tabular}




\subsection{ECCM performance}

In the simulation, it is assumed that the jammer can intercept the transmitted signal without any loss. The jammer intercepts the first radar pulse signal for circular forwarding, and the jammer intercepts the first radar pulse signal; for one-by-one forwarding, the jammer intercepts the signal and lags one PRI for transmitting signal; for the broadband forwarding, the bandwidth of jamming signal can completely cover the bandwidth of transmitted signal. The ECCM improvement factor (EIF) is applied for ECCM performance evaluation, which is defined by

$$
I_{\mathrm{EIF}}=\frac{(S / J)_{o}}{(S / J)_{i}}
$$

where $(S / J)_{o}$ is the radar output SJR after ECCM scheme is adopted; $\left(S / J_{i}\right.$ is radar output SJR without the application of any ECCM method.

Fig.5plots the range-Doppler domain output in condition of DRFM jammer working in circular forwarding mode. It can be observed from Fig.5(a)that multi-false targets jamming covers both range and Doppler dimension, and real target echoes have been completely suppressed the jamming power; Fig. 5 (b) and Fig. 5 (c) are the range-Doppler images after using the CBFM-WOD and FSVLFM-WOD ECCM methods. It shows that most of jamming power is eliminated, and CBFM-WOD is better than FSVLFM-WOD in circular forwarding mode.
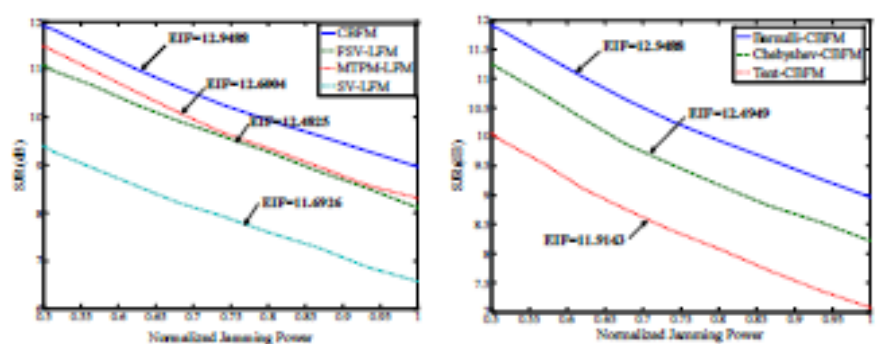

(a) Comparison of the orthogonal signals (b) Comparison of the CBFM signals

Fig. 5.Range-Doppler images in circular forwarding mode

Fig.6shows the different orthogonal diversity signal ECCM performance on SJR and EIF in circular forwarding mode. Fig.6 (a) compares the orthogonal signals of CBFM, FSV-LFM, MTPM-LFM and SV-LFM. It is obvious that CBFM signal has the most optimal ECCM performance in circular forwarding mode. Fig.6 (b) compares the three mapping CBFM signals of Bernoulli, Chebyshev and Tent. 

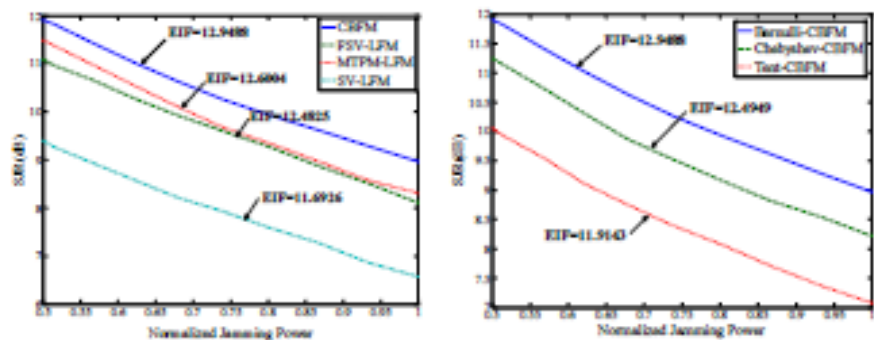

(a) Comparison of the orthogonal signals (b) Comparison of the CBFM signals Fig. 6.SJR versus jamming power

Fig.7 plots the range-Doppler domain output in condition of DRFM jammer working in one-by-one forwarding mode. It can be observed from Fig.7(a) that the multi-false targets jammingcovers both rangeand Doppler dimension. Besides, the real target echoes has been completely suppressed by the jamming power. Fig.7 (b) and (c) are range-Doppler images after CBFM-WOD and FSVLFM-WOD are adopted.It shows that most of the jamming power is eliminated, and FSVLFM-WOD is better than CBFM -WOD in one-by-one forwarding mode.

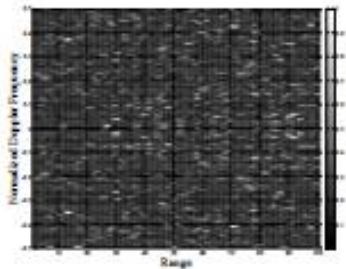

(a) No ECCM method is adopted

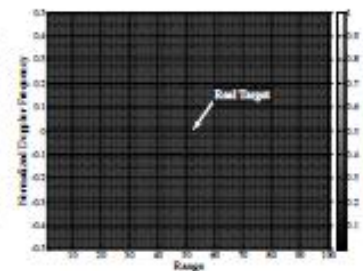

(b) CBFM-WOD

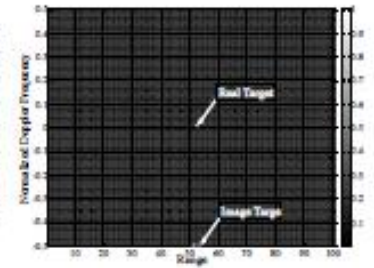

(c) FSVLFM-WOD Fig. $\overline{7}$. Range-Doppler image in one-by-one forwarding mode

Fig.8shows the different orthogonal diversity signal ECCM performance in one-by-one forwarding mode. Fig.8 (a) compares the four orthogonal signals of CBFM, FSV-LFM, MTPM-LFM and SV-LFM. Obviously, FSV-LFM signal can completely map the jammingpower outside the range gate, and it has the best ECCM performance. However, there is the vice frequency peak due to pulse signal parameter changes during pulse diversity; Fig.8 (b) compares three mapping CBFM signals of Bernoulli, Chebyshev and Tent. 

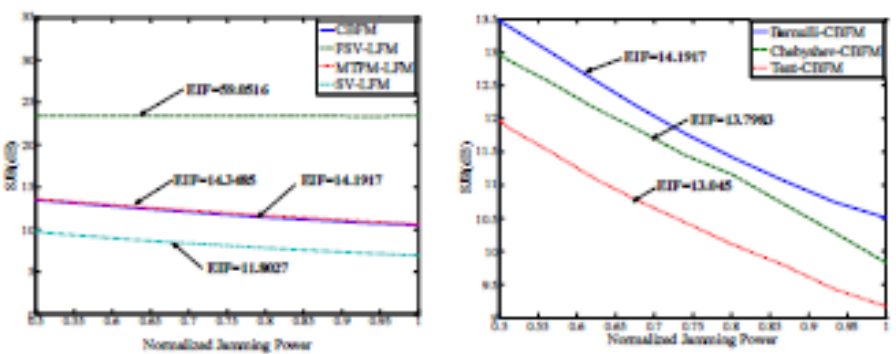

(a) Comparison of the orthogonal signals (b) Comparison of the CBFM signals Fig. 8.SJR versus jamming power

Fig.9plots the range-Doppler domain output in broadband forwarding mode. Fig.9 (b) and (c) are the result after CBFM-WOD and FSVLFM-WOD methods are adopted. It can be seen that partial jammingpower is eliminated after CBFMWOD is adopted. Through using FSVLFM-WOD, only a small part of jamming power is eliminated.

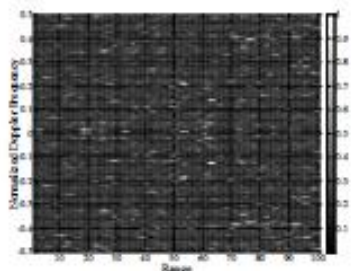

(a) No ECCM method is adopted

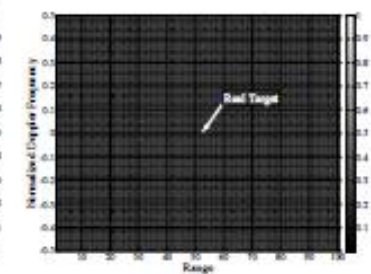

(b) CBFM-WOD

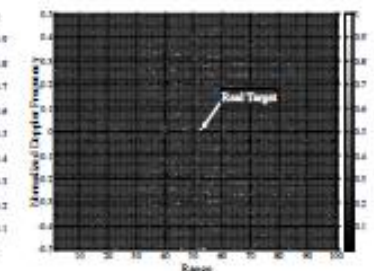

(c) FSVLFM-WOD Fig. 9.Range-Doppler image in broadband forwarding mode

Fig.10 show the different orthogonal diversity signal ECCM performance in broadband forwarding mode. Fig.10 (a) compares the four orthogonal signals of CBFM, FSV-LFM, MTPM-LFM and SV-LFM. It can be seen that the CBFM signal has been possessed with the bestECCM performance, and FSVLFM is obviously not suitable for broadband forwarding mode; Fig.10 (b) compares three mapping CBFM signals of Bernoulli, Chebyshev and Tent.
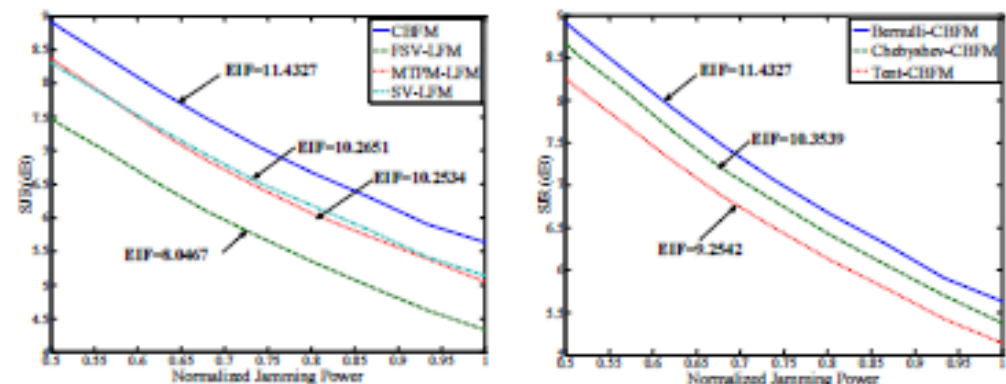

(a) Comparison of the orthogonal signals (b) Comparison of theCBFM signals (a) Comparison of the orthogonal signals (b) Comparison of theCBFM signals Fig. 10.SJR versus jamming power 


\section{Conclusions}

To conclude, in this work, an ECCM scheme is proposed based on the theory of orthogonal diversity through using two kinds of orthogonal signals for multifalse targets jamming to obtain the following conclusions:

(1) CBFM-WOD and FSVLFM-WOD signals have the ability to confront multi-false targets jamming. However, for different ways of jamming mode, there are differences in ECCM performance, and the confrontation can be conducted through selecting the superiority of adaptive diversity techniques.

(2) CBFM-WOD is suitable for confronting multi-false targets jamming of circular and broadband forwarding mode, and the FSVLFM-WOD is suitable for one-by-one forwarding mode of DRFM jammer.

(3) When selecting CBFM signals, Bernoulli CBFM has the best ECCM performance, followed by is Chebyshev CBFM. In addition, the selection of the chaotic mapping parameter $\mu$ also has some impact on the ECCM performance. As a result, is necessary to select the appropriate parameters to guarantee the ECCM performance.

\section{References}

1. Olivier K, Cilliers J E, Plessis M. Design and performance ofwideband DRFM for radar test and evaluation[J]. Electronics Letters, 2011, 37(14): 824-825.

2. Yuan L, Gaohuan L, Huilian C. A new technology of multi-false targets deception against chirp waveform inverse synthetic aperture $\operatorname{radar}[\mathrm{C}] / /$ International Conference on Signal Processing. 2008:2477-2480.

3. Li Y, Luo X M, Lv G H. The study of multi-false targets synthesizing technology against chirp ISAR[C]// International Conference on Microwave and Millimeter Wave Technology. 2008:802-805.

4. AkhtarJ. Orthogonal block coded ECCM schemes against repeat radar jammers[J]. IEEE Trans. on Aeros. Electron. Sys., 2009, 45(3): 1218-1226.

5. SoumekhM, Buffalo S. SAR-ECCM using phase-perturbed LFM chirp signals and DRFM repeat jammer penalization[J]. IEEE Trans. on Aerospace and Electronic System, 2006, 42(1): 191-205.

6. JabranA. An ECCM scheme for orthogonal independent range-focusing of real and false targets[C]//Radar Conference, 2007: 846-849.

7. CallegariS, Rovatti R. Spectral properties of chaos-based FM signals: theory and simulation results[J]. IEEE trans. on Circuits and System: Fundamental Theory and Applications, 2003, 50(1): 3-15. 